\title{
From molecular machines to macroscopic fields: an ac- cent to characteristic times
}

\author{
Lev V. Beloussov, Ilya V. Volodyaev* \\ Laboratory of Developmental Biophysics, Faculty of Biology, Moscow State University, Moscow, 119991, Russia
}

Email address:

morphogenesis@yandex.ru (L. V. Beloussov), ivolodyaev@gmail.com (I. V. Volodyaev)

To cite this article:

Lev V. Beloussov, Ilya V. Volodyaev. From Molecular Machines to Macroscopic Fields: an Accent to Characteristic Times, European Journal of Biophysics. Vol. 1, No. 1, 2013, pp. 6-15. doi: 10.11648/j.ejb.20130101.12

\begin{abstract}
The aim of this essay is to review up to now scattered data related to the integration of activities of molecular machines into higher level events, providing macroscopic organization of living beings. According to the self-organization theory, one of the main instruments for such integration is enlarging of characteristic times, going as a rule, hand by hand with characteristic dimensions. We start from the "classical", short-range mechanoelectrical protein machines emphasizing their capacity to slow down the rate of energy relaxation and to concentrate energy in a restricted number of freedom degrees. Then we pass to newly described "low entropy machines", and briefly discuss characteristic times of morphogenetic processes. A special emphasis is made on temporal patterns of the so called biophoton emission (ultraweak photon emission from the living organisms) which have a remarkable property to be spread throughout the levels ranging from milliseconds to dozens of minutes.
\end{abstract}

Keywords: Molecular machines; Self-organization; Characteristic times; Ultraweak photon emission; Fourier spectra

\section{Introduction}

All the living beings are known to be far from thermodynamic equilibrium, using this non-equilibrium for their purposes and discharging into the outer space measurable amounts of mechanical, electrostatic and electromagnetic energy. Within several last decades a substantial progress was achieved in revealing molecular mechanisms, involved in the generation and transformations of these kinds of energy within living matter. On the other hand, a simple question remains unsolved up to now - how these micro-machines, with characteristic dimensions of about several nanometers and oscillation periods within $10^{11} \mathrm{~Hz}$ range, integrate their activities for providing a coordinated behavior of macroscopic organisms whose linear and temporal dimensions are more than a dozen orders greater?

The aim of this essay is just to attract the interest of investigators to this fundamental problem, rather than to suggest its solution. We would like to start from bridging the gap between our knowledge of molecular generators and transformers of energy, and some biological and physical concepts dealing with macroscopic order of entire organisms. These concepts include the idea of morphogenetic fields [1-4] on the one hand, and the self-organization theory, SOT [5-9] on the other hand. The concept of mor- phogenetic fields postulates that developing organism is a system of position-dependent forces, regulated from the upper levels and molding successive embryonic shapes. Remarkably, in the latest version of this theory, its founder, Russian biologist Alexander Gurwitsch, regarded molecules as objects of field action, defining field as a factor vectorizing a certain part of molecular excitation energy [1]. This makes it reasonable to discuss relations of this theory with the modern concept of so called protein-machines.

On the other hand, SOT is a well known and perfectly elaborated theory using qualitative analysis of non-linear differential equations for explaining how a new macroscopic scale can be generated on the basis of much more local interactions, possessing no linear scale at all [9]. An important property of SOT (lacking in the classical morphogenetic field theories) is an accent to the hierarchy of characteristic times (Tch). It is claimed indeed $[6,7]$ that processes with greater Tch can "slave" more fast ones, providing thus the emergence of not only temporal, but also spatial organization of higher levels. Accordingly, the aim of this review will be to put together some as yet quite scattered and non-complete data about the different levels Tch and their capacities to shift from smaller to greater ones. We shall start from the so called "protein machines", that is, elementary transformers of chemical potentials to 
mechanical, electrostatic and electromagnetic energy, and distinguish two types of such machines: individual protein molecules, froming "classical", or short range machines, and recently discovered low entropy machines, based upon coherent behavior of large ensembles of water molecules. Then we'll make a jump towards morphogenetic processes of much greater Tch. At last, we come to a peculiar group of "biophotonic" events which, under proper analysis give a hope to bring together events of quite different characteristic times.

\section{Classical (Short Range) Pro- tein-Machines}

By text-book definitions [10] a protein machine consists of a molecule possessing ATPase activity, a fibril along which this molecule is translocated and a load which it transports. This definition fits such constructions as actomyosin and other microfilament and microtubule associated motors, but it is too narrow and, most important, does not elucidate the basic physical principles of these devices. More broad definition embraces a wider class of protein-machines, which transform non-vectorized chemical energy into vectorized mechanical one, even if they do not bear any load and do not split ATP molecule at the given time moment. Several decades ago such devices got a remarkable name of "Brownian ratchets" [11] meaning that they transform chaotic (Brownian) molecular movement into directed one. Actin microfilaments, pushing the leading edge of crawling fibroblast, were often taken as an example. Subsequent investigations demonstrated however, that these "ratchets" are organized in much more complicated way than it had been suggested before. Most important role is played by a dimeric protein, formin, located at the +end of the double helical microfilament, and oscillating between $\backslash$ screwed and relaxed states with about $10 \mathrm{~Hz}$ frequency. As a result, the torsion energy of about $1 \mathrm{eV}$ is periodically accumulated and discharged. This energy is equivalent to that released by splitting of about 10 ATP molecules. However, in this process no such splitting is taking place: by a model suggested [12] all what we have here is the exchange of elastic energy between formin and adjacent microfilament subunits, with some gain got due to establishment of hydrophobic bonds between the newly inserted actin subunits.

The main common feature of all the protein-machines is considerable slowdown of relaxation of the accumulated energy, and enormous restriction (often up to one) in the number of degrees of freedom onto which the relaxation is taking place [13-15]. At the same time, amplitudes of the directed movements performed along the rested freedom degrees reach characteristic dimensions of the machine itself (which is impossible in the case of man-made industrial machines). These properties are provided by a specific structure of protein macromolecules, which include:

- Relatively soft elastic spring accumulating energy in the form of elastic stresses. The spring consists of non-spiral parts and electrically charged groups;

- Rigid "levers" which transmit stresses ( $\alpha$-spirals);

- Hinge joint-points, represented by small aminoacids, for example glycine;

- Fixation points: hydrogen bonds, S-S bonds.

The energy accumulated in elastic elements is estimated as $\sim 0,5 \mathrm{eV}$ per $5 \times 10^{-9} \mathrm{~m}$ (length of the element), while the lifetime of the metastable mechanically stressed states ranges between $10^{-6}$ and $10^{3} \mathrm{~s}$, which exceeds self-frequencies of protein molecules $\left(10^{11}-10^{12} \mathrm{~s}^{-1}\right)$ by about 15 orders [15]!

Thus, the main and tightly interconnected properties of protein-machines are:

- Greatly retarded relaxation of accumulated elastic energy, producing quite large (in the macromolecular scale) and essentially vectorized shifts;

- Coexistence and practical indivisibility of mechanical and electrostatic components, permitting to define these machines as electro-mechanical.

Let us comment the latter point. Electrically charged groups (mostly negatively charged carbonyls and positively charged aminoacids) contribute to maintenance of mechanically stressed state of protein macromolecules. Same role should be played by charged ligands, in particular ATP, containing three negatively charged phosphate groups. Therefore, binding ATP to proteins should increase electrostatic repulsion onto the latter's surface and hence straighten the protein molecules, supplying them by additional elastic energy. By Pollack's estimation [16], this elastic energy is two times greater than energy provided by ATP molecule splitting. Taking into consideration, that each next step of practically all the signaling pathways is associated with phosphorylation of proteins, this kind of molecular deformation should play a great, however up to now practically neglected role in cell signaling.

Until now we were dealing with chemo- electro- mechanical transformers in the form of isolated macromolecules (for example enzymes) acting in their immediate vicinity (non-exceeding the radii of molecular interactions). Meanwhile, the discussed example of ATP-protein interactions led some authors to postulate existence of a quite different class of machines, characterized by highly extended range of action, including billions of uniformly oriented water molecules. These will be described in the next section.

\section{Low Entropy Machines}

In his "association-induction" theory Ling [17] points out that an ATP molecule adsorbed by a protein, is a powerful electron acceptor, reducing the electron density onto the protein surface within considerable distances. As a consequence, "the liberated carboxyl groups start to absorb $\mathrm{K}^{+}$while $\mathrm{NH}_{2}^{-}$and $\mathrm{CO}^{-}$groups liberated from participating in creating secondary structures start to interact with water, forming a multilayer structure consisting of polarized and 
uniformly oriented water structures" [18].

This is one among several scenarios of formation of low entropy water domains. As theoretically predicted [18, 19], if the density of any ensemble of microscopic particles exceeds a certain threshold, the particles start to oscillate in a coherent way. Such "coherent water domains" (CWD) may extend up to $100 \mathrm{~nm}$ and consist of about $10^{7}$ water molecules which oscillate with $10^{11} \mathrm{~Hz}$ frequency. Due to a high degree of order, CWD are the areas of very low entropy and are thus able to absorb non-coherent (heat) energy from the surrounding bulk water and transform it into a "high grade" coherent one. Noteworthy, CWD absorb light in infrared and UV spectral regions. The absorbed energy may reach $12,06 \mathrm{eV}$ which is only in $\sim 0,5 \mathrm{eV}$ lower than the energy of water ionization. In other words, CWD possess a substantial amount of free or almost free electrons, which results in the electrical potential difference between CWD and bulk water. In such a way, low entropy areas are real machines, producing electrical energy. Also, they are similar to macromolecular machines described above (as well as to photonic machines - to be described below) by providing electron excitation with long lifetime. Moreover, these machines are postulated to produce direct mechanical effects by bringing together molecules oscillating in unison with each other.

What might be the biological role of such machines and, in particular, of coherent state of water domains? This is as yet unanswered question of basic importance. Del Giudice et al. [18] speculate that such a state is important for providing extreme temperature stability within living systems, avoidance of Joule effects during ions traffic and, most important, precise meeting of partner molecules during enzymatic reactions. Sensitivity of living matter to microwaves can be also associated with the presence of CWD [19]. Here we are confronted with a rare for biology situation where theoretical predictions are ahead of experimenting, and a real challenge to lab biologists is to do everything possible for exploring the role of low entropy machines, because this could revolutionize entire image of cell biology.

\section{Temporal Structure of Biophoton Emission}

Ultraweak photon emission (UPE) from living organisms was discovered almost 90 years ago and its existence has been confirmed in a great number of studies since then (recent reviews: [20-22]), although views upon its biological role and physico-chemical mechanisms of photons generation remain quite diverse. Two main different (but not necessarily mutually exclusive) theories upon UPE origin are the following. According to the first theory, an impulse of UPE is produced in a single step due to recombination of free radicals which are the by-products of many chemical reactions, mostly oxidative. According to the second view point, photonic energy level (several electron-volts) may be reached by summation of smaller portions of energy released during collective molecular interactions in highly ordered dense medium. The second scenario should be favored on the one hand by slowing down relaxation of energy accumulated in molecules and supramolecular structures and, on the other hand by increasing space-temporal density of energy-producing events. The first of these events has been already discussed as belonging to the main properties of both short- and long-range molecular machines. Probability of the second condition was discussed by Slawinsky who gave in [20] several relevant examples. Thus, relaxation of 9 supertwists in DNA molecule liberates $2 \mathrm{eV}$ in $0,01 \mathrm{~s}$; enzymatic splitting of acetylcholine at the postsynaptic membrane releases up to $1,2 \mathrm{eV}$ in even much shorter time period $(4 \times 10-7 \mathrm{~s})$. In both cases, released energy (which corresponds to visible spectral range) has good chances to avoid dissipation.

Similar estimations may be applied to cytoskeletal structures. Thus, by Pokorny [23] , a single microtubule stores power of $\sim 10-14 \mathrm{~W} / \mathrm{cm}$, obtained due to rapid post-polymerization GTP hydrolysis. Taking an average microtubule length of about $10 \mu \mathrm{m}$, and assuming that no less than 100 microtubules per second are polymerized in close vicinity, power of about $10-15 \mathrm{~W}$ is produced in a proper proximity, and thus is potentially able to avoid dissipation and sum up to photonic level. This amount of energy exceeds the power of a photon emission sensed by a standard photomultiplier, by two orders. And the last but not the least, photonic level energy can be accumulated in coherent water domains described above.

In this context an almost unexplored problem of temporal organization of UPE may be of considerable interest. First, if the above presented concepts are at least partly true, one should expect that UPE consists of brief bursts (discharges) separated by more prolonged periods of energy accumulation. Also, destruction or damage of structures, expected to be involved in energy accumulation, should result in more extensive bursts. Last, by measuring intervals between bursts and exploring whether there is any temporal order in their successions, one may hope to get unique information about the scales of structures involved in energy accumulation up to photonic level.

Here we give a brief account of our UPE measurements from Xenopus and loach (Misgurnus fossilis) embryos and monolayer cell cultures, obtained within two last decades in the International Institute of Biophysics (Neuss, Germany). A detailed account of the data obtained (with the exception of measurements performed on Xenopus embryos) and the description of the measurement technique have been presented elsewhere [24-26]. In all the cases what we define as adequate controls to the samples' measurements were UPE records from similar quartz cuvettes with the same medium.

\subsection{UPE Consists of Single Bursts of No More Than Mil- liseconds Duration Separated by Prolonged Pauses}

Detailed measurements using millisecond dwell times 
were performed on Xenopus eggs and embryos. Significant excesses over control data were detected in parthenogenetically activated, just fertilized and early cleaving eggs, as well as in midblastula and early neurula stage embryos. Remarkably, extensive UPE from midblastula stage embryos coincided with a burst of activation of embryonic genes and start of RNA synthesis. In all the cases UPE consisted of single flashes of no more than $10^{-3} \mathrm{~s}$ duration each (Fig.1, 2). Altogether they occupied less than 1\% of the total measurement time and were separated by pauses lasting from $\sim 5$ to $100 \mathrm{msec}$. Continuous groups of no more than two neighboring flashes were quite rare (covering less than $5 \%$ of the total number of flashes). However, some more loose aggregations of flashes could be numerously seen in developing samples (but never in background counts) (Fig. 1A, E, 2C). In the total, from 80 to $90 \%$ of flashes were of the minimal values (one count $/ \mathrm{msec}$ ), while the amounts of greater counts have been exponentially reduced, although single 40-60 counts/msec flashes could be traced both in developing samples and background UPE. More prolonged records showed peculiar constellations of great flashes (Fig. 1B, 2B, D) pointing to some kind of long range temporal order, to be discussed below in more details. Similar UPE patterns consisting of solitary milliseconds flashes took place in loach embryos and cell cultures.
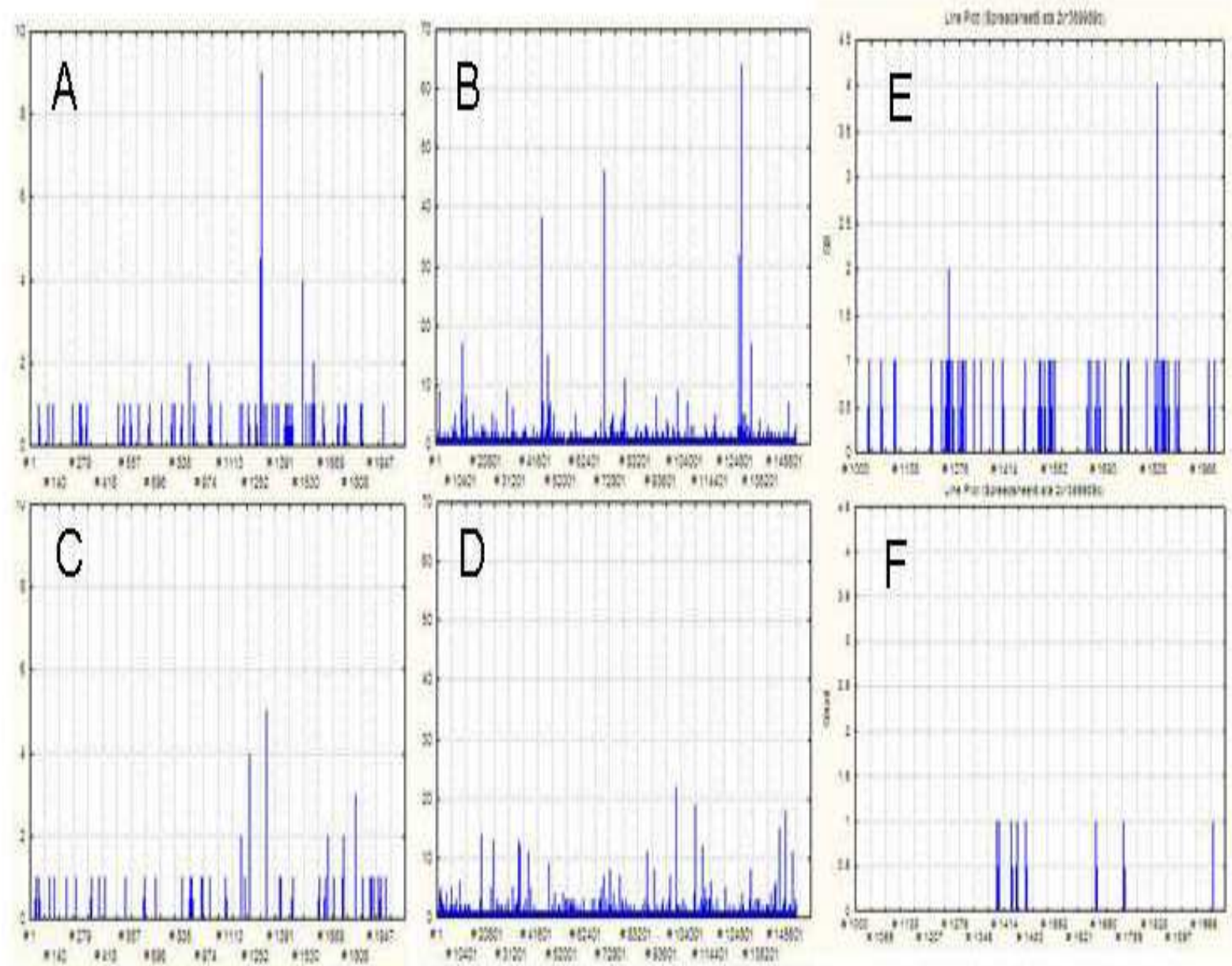

Figure 1. Records of photon emission from the early cleavage (A, B) and midblastula stage Xenopus embryos (E) compared to adequate background counts $(C, D, F)$. A, C, E, F: 2 seconds duration, $B, D: 2,5$ min duration records. Dwell time 1 msec. Note aggregation of flashes accompanied by emission bursts in $A$ and an ordered series of extensive bursts in $B$. 

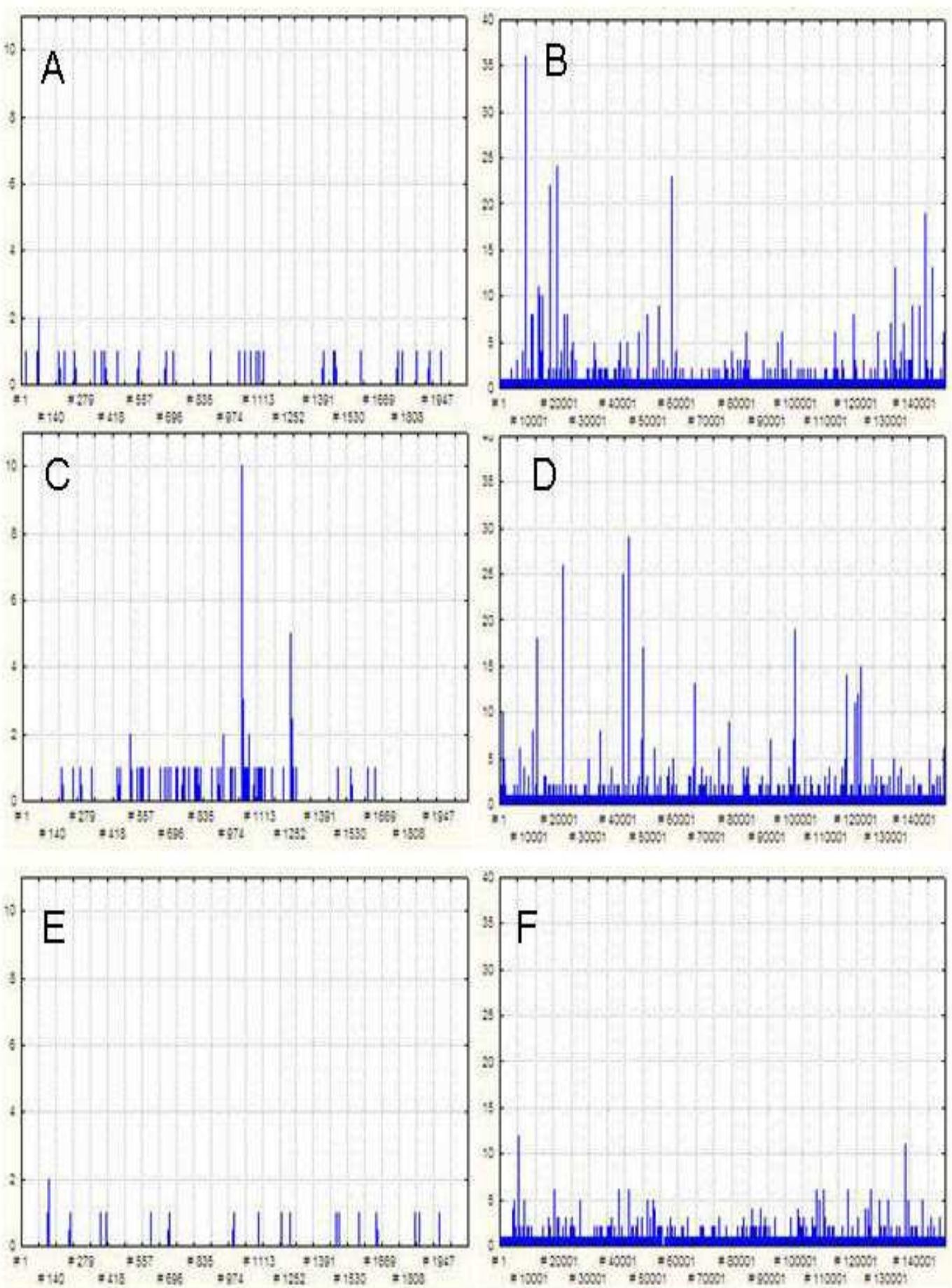

Figure 2. Photon emission patterns of early neurula Xenopus embryos (A-D) compared to background counts (E-F). Left column: 2 s, right column: 2,5 min duration records. A, B: records from a single embryo. C, D: records from 5 embryos. E, F: background counts. Dwell times 1 msec. Note aggregation of flashes accompanied by emission bursts in $C$ and ordered series of extensive bursts in $B, D$.

\subsection{More Obvious Temporal Order Appears at Higher Tch}

By using greater dwell times and enlarging correspondingly the measurements periods, more prolonged regular UPE oscillations can be traced (Fig. 3). Thus, midblastula stage Xenopus embryos demonstrate quite pronounced 20
$\mathrm{Hz}$ spectral density peak at $0,01 \mathrm{~s}$ dwell time, and several additional peaks of smaller frequencies at 0,1 and $1 \mathrm{~s}$ dwells (as shown in Fig. 3A). Stage-specific Fourier spectra of UPE were also quite pronounced in loach embryos (Fig. $3 \mathrm{~B}, \mathrm{C})$. About $10^{-1} \mathrm{~Hz}$ oscillations were mostly regular during eggs cleavage and were exchanged at the latest stages by more complicated patterns. 


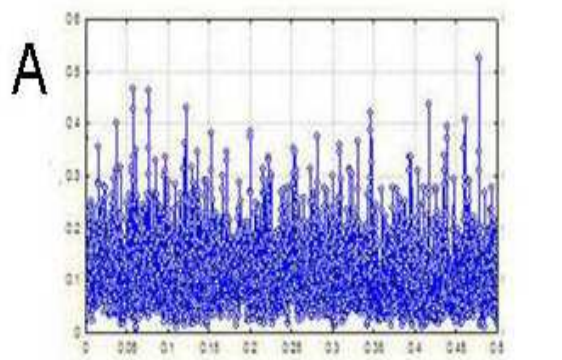

\section{B}
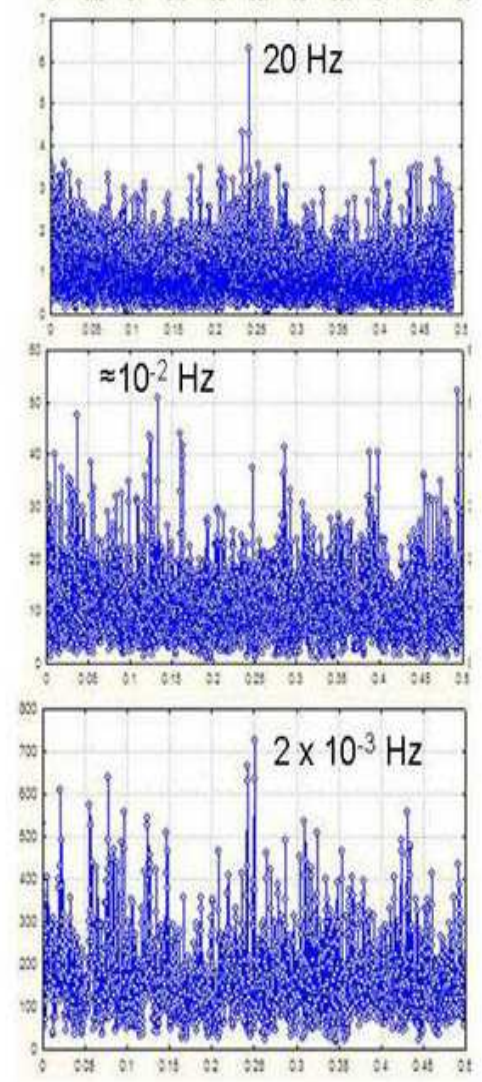
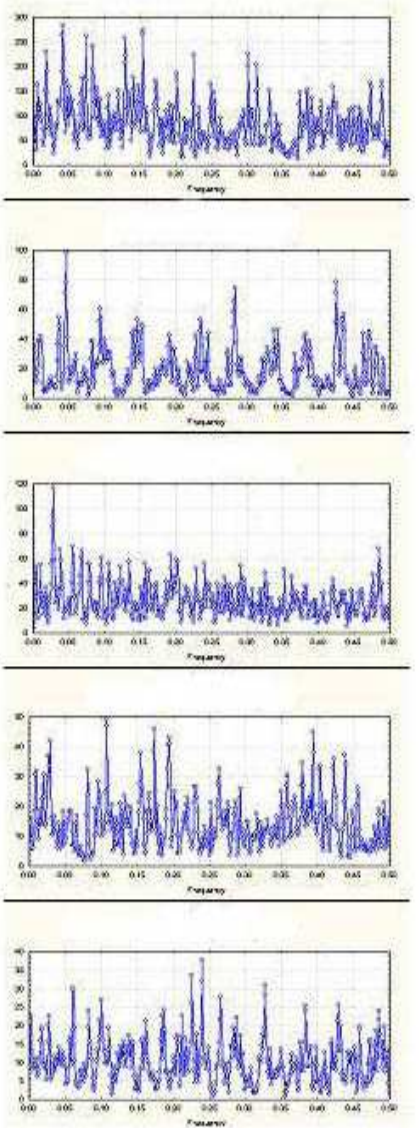

C
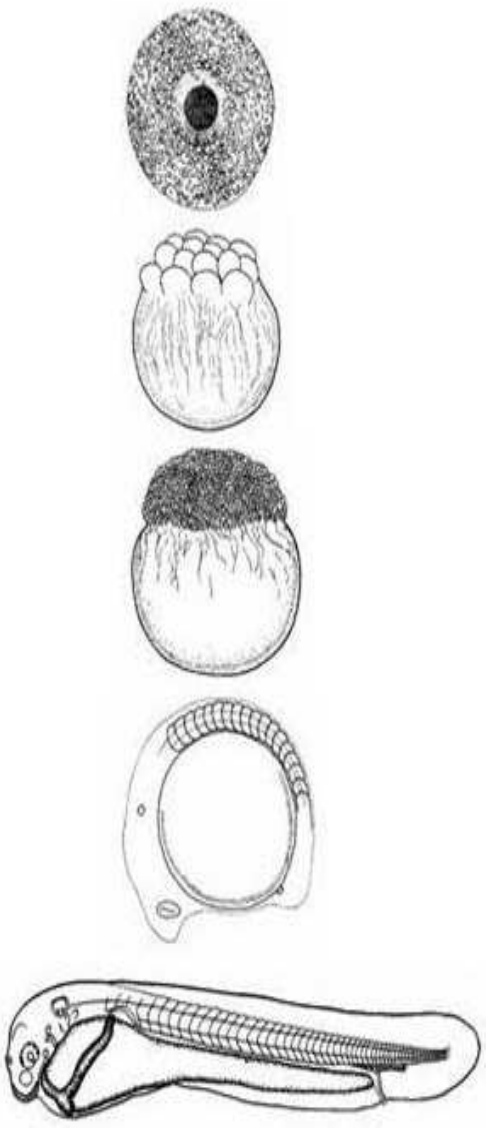

Figure 3. Fourier spectra (in terms of spectral densities) from midblastula stage Xenopus embryos (A) and Misgurnus fossilis embryos (B) at the successive developmental stages shown in $C$. In A dwell times from up to down are 0,001, 0,01, 0,1 and 1 sec. Correspondingly the measuring time intervals are 8, 80, 800 and $8000 \mathrm{~s}$. Frequencies of the most outstanding peaks are shown. Measurements shown in B cover 2 min periods and are presented as autocorellograms of Fourier spectra.

\subsection{Activation of Signaling Pathways, Destruction of Cy- toskeletal Structures and Non-Specific Stresses In- duce Periodic UPE Patterns}

By adding $10^{-9} \mathrm{~g} / \mathrm{ml}$ of FGF-1 (fibroblast growth factor) to a monolayer culture (consisting of about $10^{5}$ cells) of mouse fibroblasts (line C3H10T1/2) mounted onto vertical wall of quartz cuvette, we observed rapid appearance of pronounced $10^{1}-10^{2} \mathrm{~s}$ range UPE oscillations (Fig. 4A). As seen by a series of autocorellograms (Fig. 4B) they distinct very much from the spectrum of non-affected culture and are gradually evolving, indicating surprising changes of oscillatory pattern during signal processing. It is of interest, that monolayer cultures of hyppocamp (mid-brain) cells reveal a similar evolution of UPE oscillatory patterns going now spontaneously, without any intervention from outside
(Fig. 4C). This may be attributed to inherent properties of brain cells.

Destruction of actin microfilaments by addition of cytochalasine D $(10 \mu \mathrm{g} / \mathrm{ml})$ to monolayer fibroblast culture also induced pronounced oscillations (Fig. 5A) which included $5 \times 10^{-1}, 7,6 \times 10^{-2}$ and $7,6 \times 10^{-3} \mathrm{~Hz}$ peaks, preserved up to 20 min. Similar peaks of $5 \times 10^{-1}, 10^{-1}, 2,5 \times 10^{-2}$ and $5 \times 10^{-3} \mathrm{~Hz}$ frequencies were induced less than $1 \mathrm{~min}$ after addition of $10^{-4} \mathrm{M}$ colchicine (agent preventing microtubules assembly) to a monolayer fibroblast culture. Quite pronounced oscillations took place for several minutes time periods, after transferring cardyomyocytes monolayer cultures from nutritive to weak medium (Fig. 5B), as well as after addition of trypsin/EDTA $(0,025 \%)$ to fibroblasts cultures. These latter responses to non-specific damaging factors can be qualified as peculiar "alarm signals" of cells. 


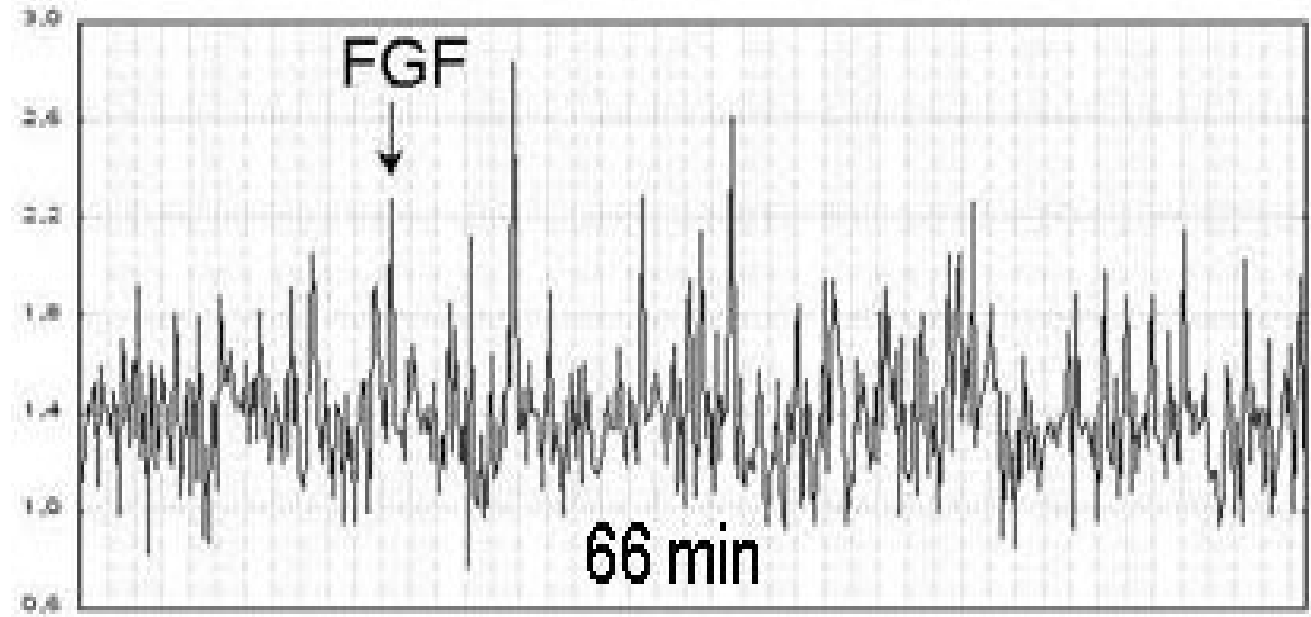

(A)
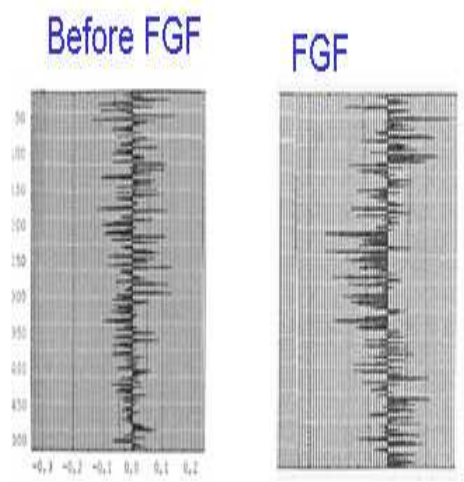

$10 \mathrm{~min}$

\section{$20 \mathrm{~min}$}

$30 \mathrm{~min}$

$40 \mathrm{~min}$
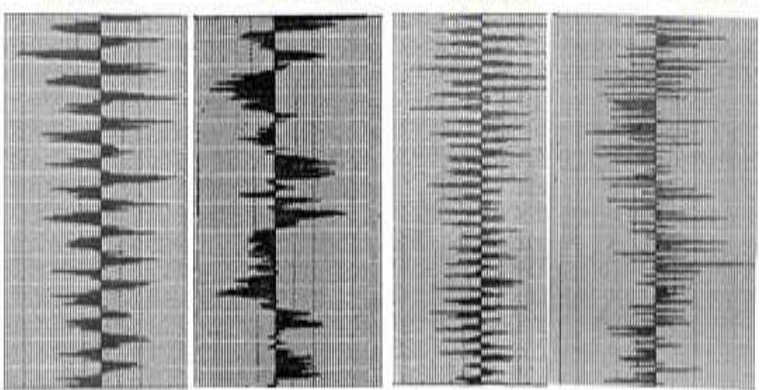

(B)

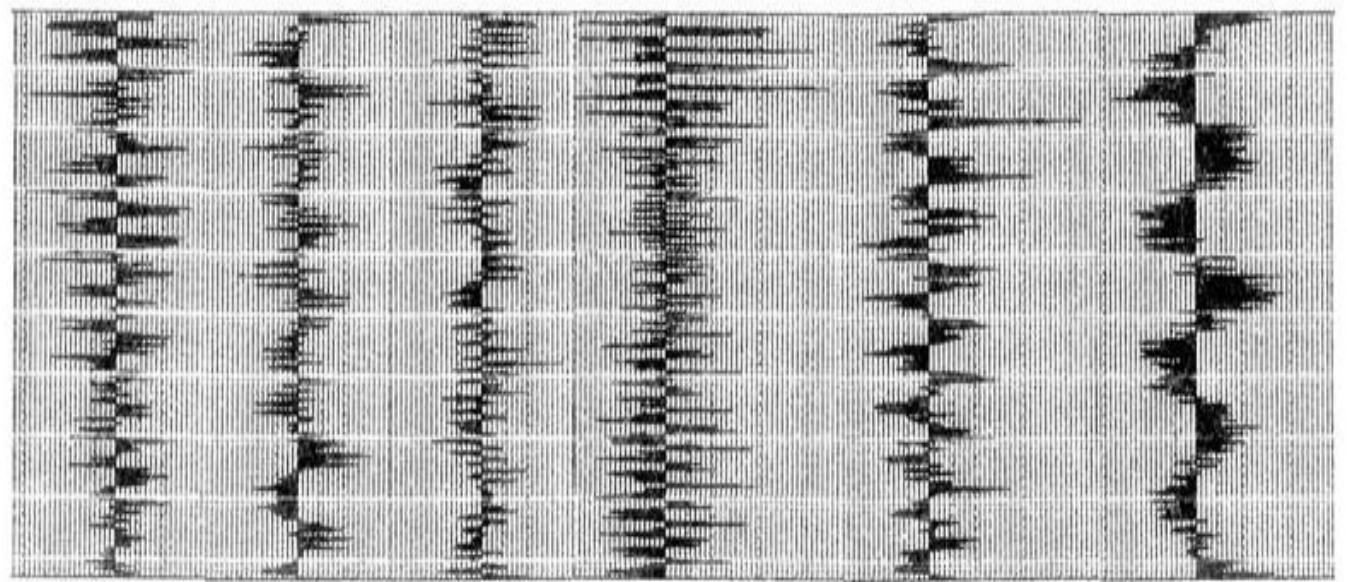

(C)

Figure 4. UPE record (A) and autocorellograms of Fourier spectra $(B, C)$ from monolayer cell cultures. $A$, $B$ : fibroblast culture under the action of $f i$ broblast growth factor (FGF-1). In A horizontal axis is time (min), vertical axis shows UPE intensity (counts/s). B: autocorellograms of UPE Fourier spectra corresponding to 5 min duration records performed under successive time intervals before and after FGF addition (shown). C: Fourier spectra (in terms of autocorellograms) from UPE of a monolayer culture of hyppocamp cells. Measurement periods were 5 min each, separated by 10 min intervals. In $B, C$ note the evolution of spectral patterns. 


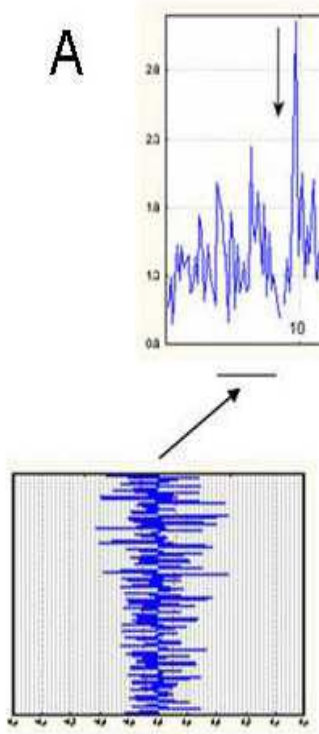

Before

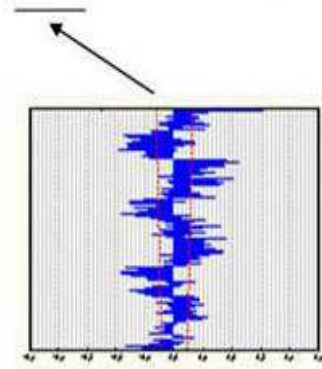

after addition
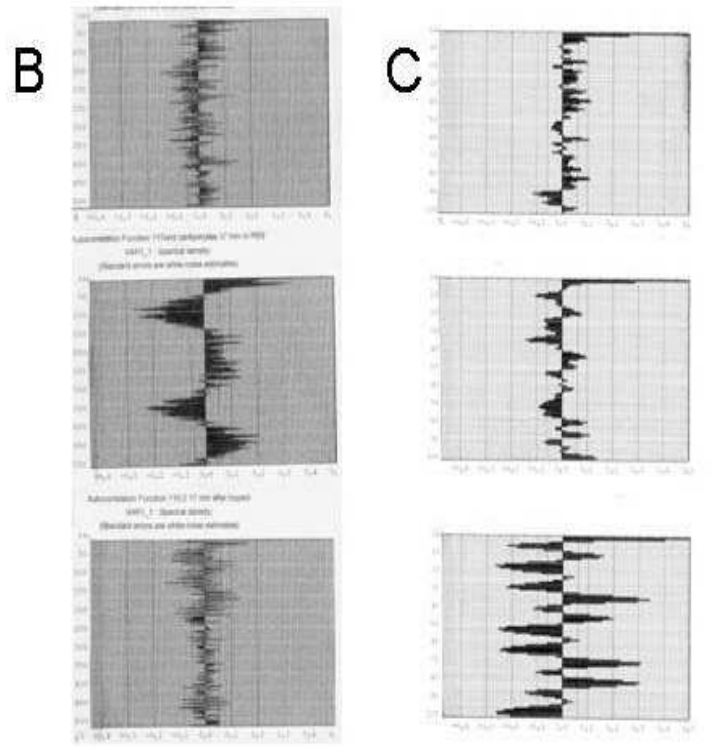

Figure 5. Effects of cytochalasine D (CD) addition and non-specific stressful factors upon UPE Fourier spectra of monolayer fibroblasts $(A, C)$ and cardyomyocytes (B) cultures. A: UPE record (vertical arrow indicates the moment of CD application) and 5 min long records of Fourier patterns before and after CD application in terms of autocorrelograms. Note increase of spectral coherency soon after CD application. B, from up to down: autocorellograms of Fourier spectra from intact cardyomyocytes incubated in nutritive medium, those incubated in hunger medium (PBS) and affected by trypsin. C, from up to down: similar spectra from intact fibroblasts, immediately addition of trypsin and 10 min later.

\section{Some Remarks on Characteristic Times of Morphogenetic Processes}

The hierarchy of Tch involved in morphogenetic processes can be best illustrated with a group of lower Invertebrates, hydroid polyps, which create branched colonies terminated by hydranths. Stems and hydranths are growing and change their shapes due to series of the so called growth pulsations (GP), each of them lasting several minutes [27]. At the beginning of each next GP several dozen cells arranged at the tip are swelled and rotate towards perpendicular orientations, producing lateral pressure on each other. As a result, the tip is extended and deformed. At the second GP phase cells are deswelled and restore the initial oblique orientation. Relative duration of extension and retraction GP phases is precisely regulated in the species- and stage-specific manner, and directly determine the shape of hydranths (their transversal/longitudinal length ratio): the greater is the relative duration of extension phase the wider are the hydranths. Moreover, not only relative duration of GP phases, but also total number of successive GPs required for stem growth or hydranths formation, are strictly programmed, determining length of the corresponding rudiments. Sums of these GPs give a next, higher order Tch. Therefore, in this group of organisms spatial organization is directly encoded by temporal hierarchy. It is still a challenge to comprehend how a complicated web of inter- and intracellular signals and vacuolar transport becomes integrated in so precise cycles with much greater Tch.

Interestingly, in the development of higher animals (Ver- tebrates), recognizable temporal modules (times of somite formation; temporal distances between drastic reconstructions in embryo topology) have even greater Tch (of several dozen minutes order) and are not underlined by any shorter more or less regular temporal cycles: large scale temporal organization (emerged hand by hand with macroscopic spatial organization) looks arising de novo, out of much more random microscopic one. Even if being unable to give a precise explanation of this feature, we can definitely attribute it to general properties of self-organizing systems, demanding parametric regulation.

\section{Dissipative and Non-Dissipative Components of Morphogenesis}

With the formulation of the dissipative structures theory (5-9), a principal step was made in bridging the gap between physics and biology. However, enthusiasm born by such a progress sometimes led to a hasty conclusion that all, or at least the main structures created during morphogenesis, are dissipative in senso stricto, that is they require a constant flow of energy for maintenance. Meanwhile, even our everyday experience, to say nothing about paleontology, argues for the opposite: even minor morphological details can be kept intact for billions of years without energy supply. Looking more carefully on morphogenetic processes, and taking again hydroid polyps as an example, we can see that real self-organization, and processes with classical, close to equilibrium dynamics, coexist and alternate each other. Growth pulsations taken per se, are definitely dissipative 
indeed, as they require energy for individual cell contractility. However, next steps leading to emergence of macroscopic shapes are not energy consuming: cells swelling, which produces tangential pressure stresses within the cell layer, is associated with a shift towards mechanical equilibrium; and the specific geometry of the rudiment is the result of relaxation of these stresses. These and other examples show us that morphogenetic processes which can be related to dissipative, are endowed mostly by temporal (rather than spatial) characteristics, while the forms themselves are created when the system is moving along relaxatory pathways. As argued in [28] such a property is common for the shape-producing "machines" of quite different levels. This is not to underestimate dissipative aspect of morphogenetic events: they are not only necessary, but should be also finely regulated both from genetic and epigenetic levels. However they alone cannot be responsible for shape formation.

In the context of the above said, a recent thermodynamically-based broad classification of shape-forming processes (related by the authors to self-assembly) should be mentioned [29]. The authors distinguish: (1) equilibrium self-assembly which forms structures that minimize free energy of the system; (2) non-equilibrium self-assembly which leads "to long-lived, kinetically trapped states, which depend on the history (e.g. processing) of the system; (3) dynamic self-assembly, just fitting the definition of dissipative structures. This approach developed also in $[30,31]$ provides morphogenetic studies by more firm and adequate physical basis than what we had before.

\section{Discussion}

As it was briefly mentioned beforehand, Alexander Gurwitsch, the founder of the morphogenetic field theory, proposed that dynamical order in living organisms is supported by a single vectorizing factor, acting in the same way in all the structural levels, from molecular to organismal. Today we can see that reality is much more complicated and fragmented. What we observe on molecular level, is a set of devices (machines) accurately collected from specific molecules (mostly proteins) which prevent chemical energy stored in molecular bonds from chaotic dissipation by transforming it into high grade (low entropy) mechanical, electrical and photonic energy. In doing this, all molecular machines (not only electromechanical, but also photon-producing), in spite of all their intrinsic differences, share the common property of retarding relaxation times and extensively reducing the number of freedom degrees for the liberated energy.

This is however only the first step which is required for extending dynamical order up to macroscopic space-temporal dimensions. For fulfilling such a task, that is, for passing from, by rough estimations, $10-8 \mathrm{~cm} / 100 \mathrm{~s}$ range (typical for supramolecular events) to macro-morphological realm $\left(10^{1} \mathrm{~cm} / 10^{3} \mathrm{~s}\right.$ range) another set of powerful "+" and "_" feedbacks between molecular/supramolecular machines should come into action, which is to be regulated by macroscopic parameters. Only under these conditions, a set of individual specific "machines" can be transformed into what we call common morphogenetic fields. In these processes temporal ordering is going hand by hand with spatial one.

Feedbacks which according to SOT can provide such macrosccopic ordering are still poorly explored. Although it cannot be neglected that they are based upon a vast manifold of molecular and supramolecular events, their detailed reductionistic exploration is not enough for outlining the structure of order parameters, making emergence of macroscopic feedbacks possible. More attention should be paid to collective interactions which may produce unexpected essentially non-linear effects. In addition, traditional SOT approaches should be enriched by the notion of coherency in the sense developed in [28, 29]: only in this context temporal order maintained over several orders of magnitude can be apprehended.

\section{Acknowledgements}

This study was supported by the Russian Fund for Fundamental Research (RFFI), grant 11-04-01-718. The authors express their deep gratitude to Prof. F.-A. Popp for his hospitality and fruitful discussions in the International Institute of Biophysics.

\section{References}

[1] Gurwitsch A G 1944 A theory of biological field (in Russian) Sovetskaya Nauka Moskva.

[2] Gilbert, S.F., Opitz, J.M. and R.A. Raff 1996. Dev. Biol. 173: 357-372.

[3] Goodwin, B. 1994. How the leopard changed its spots. Weidenfeld \& Nicolson, London.

[4] Levine, M. 2012. BioSystems, 109: 243-261.

[5] Prigogine, I. 1980. From being to becoming. Freeman and Co, N.Y.

[6] Haken, H. 1978. Synergetics. Springer-Verlag Berlin Heidelberg New York.

[7] Winfree, A.T. 1987. The timing of biological clocks. Scientific American Library New York.

[8] Ball P. 1999. The self-made tapestry. Oxford Univ. Press, Oxford.

[9] Krinsky V I Zhabotinsly A M 1981 (in Russian). In: Autowave Processes in Diffusional Systems (M.T.Grechova ed.) Gorky Inst. Appl. Phys. Acad. Sci. USSR 6.

[10] Alberts R et al 2003 Molecular Biology of the Cell Garland Science Taylor and Francis Group

[11] Hanggi P Marchesoni F 2009 Rev. Mod. Phys. 81387

[12] Shemesh T Otomo T Rosen M K Bershadsky A D and M.M.Kozlov 2005 J. Cell Biol. 170889. 
[13] McClare C W F 1971 J. Theor. Biol. 301

[14] Blumenfeld L A 1983 Physics of Bioenergetic Processes. Berlin Springer

[15] Chernavskii D S Chernavskaia N M 1999 Protein-machine. Biological macromolecular constructions. Moscow University Press. (in Russian)

[16] Pollack G H 2001. Cells, Gels and the Engines of Life: A New, Unifying Approach to Cell Function. Seattle WA Ebner \& Sons.

[17] Ling G N 2001 Life at the Cell and Below-Cell Level. Pacific Press N Y

[18] Kurcz A. et al. 2010. Phys. Rev. A 81: 063821

[19] Del Giudice E. and Giuliani L. 2010 Eur. J. Oncology Lab 5

[20] Slawinski J 1988 Experientia 44559.

[21] Trushin M V 2004 Rivista di Biologia/Biology Forum 97 399.

[22] Cifra M Fields J Z Farhadi A. 2011 Progress in Biophys. Mol. Biol. 105223.
[23] Pokorny J 2006 In: Herbert Frohlich FRS: A Physicist Ahead of his Time. Univ. of Liverpool 177.

[24] Beloussov L V, Luchinskaia N N 1998 In: Biophotons (Jiin-Ju Chang, J Fisch and F.-A. Popp eds). Kluwer Acad. Publishers. Dordrecht /Boston/ London 121.

[25] Beloussov L V 2002 BioSystems 68199.

[26] Beloussov L V 2006 In: Biophotonics and Coherent Systems in Biology (L V Beloussov, V L Voeikov and V S Martynyuk eds). Springer. N Y 139.

[27] Beloussov L V, Kazakova N I, Luchinskaia N N, Novoselov V V 1997. Int. J. Devel Biol. 41793.

[28] Igamberdiev A U 2012 BioSystems 109: 336-

[29] Cademartiri L, Bishop K J M, Snyder P.W and Ozin G.A. 2012 Phil. Trans. R. Soc. A 370: 2824-2847.

[30] Grzybovski B A, Wilmer C E, Kim J, Browne K P and Bishop K J M 2009. Soft Matter 5: 1110-1128.

[31] Jarzynski C 2008 Eur. Phys. J. B: 331-340.

[32] Popp, F.-A. 1992 In: Recent Advances in Biophoton Research and its Applications (F.-A. Popp, K.H.Li and Q.Gu eds) World Scientific 1-42. 